\title{
Efficacy of Antimicrobial Lock Solutionsagainst Catheter-Related Infections in Hemodialysis Patients
}

\author{
Maria Skoulatou ${ }^{1}$, Victoria Alikari², Georgia Toulia ${ }^{2}$, Anna Kavga ${ }^{2}$, Ourania Govina ${ }^{2}$, Dimitra Siopi ${ }^{3}$, \\ Irene Zormba ${ }^{3}$, Christina Skoulatou ${ }^{4} \&$ Georgia Gerogianni $^{2}$ \\ ${ }^{1}$ Department ofNephrology, General Hospital - Health Centre of Naxos, Greece \\ ${ }^{2}$ Department of Nursing, University of West Attica, Athens, Greece \\ ${ }^{3}$ Department of Nephrology, Attikon University Hospital, Athens, Greece \\ ${ }^{4}$ Department of Microbiology, General Hospital-Health Centre of Naxos, Greece \\ Correspondence: Georgia Gerogianni, Department of Nursing, University of West Attica, Athens, Greece
}

Received: January 18, 2021 Accepted: February 26, 2021 Online Published: March 5, 2021

doi:10.5539/gjhs.v13n4p72 URL: https://doi.org/10.5539/gjhs.v13n4p72

\begin{abstract}
Introduction: Hemodialysis patientsare at increased risk of catheter-related bloodstream infections (CRBSI) and catheter thrombosis asdialysis requires regular access to blood circulation through catheters. Antimicrobial Locking Solutions (ALS) containing anticoagulants and antimicrobial agents (antibiotic/non-antibiotic) are used to seal catheters in order to prevent thrombosis and CRBSI.
\end{abstract}

Purpose: The investigation of internationalclinical studies regarding the efficacy and safety of the various ALS treatments for CRBSI in hemodialysis patients (HD patients) comparing to conventional approaches such as heparin treatment or catheter removal.

Methodology: The review included prospective or retrospective clinical studies, randomized clinical trials or cohort studies published since 2015. Publications were retrieved from the "Pubmed", "Google Scholar" and "Elsevier" databases using 'catheter', 'catheter removal', 'hemodialysis' 'bacteremia', 'antimicrobial lock therapy'as key words.

Results: 17 articles were found to meet the criteria for this systematic review. These studies investigated locking solutions containing various antibiotic (Cefazolin, Gentamicin, Vancomycin, Cotrimoxazole, Daptomycin, Trimethoprim) or/and non-antibiotic agents (Citrate, taurolidine, ethanol, urokinase, Cathasept) +/- anticoagulants, single or in various combinations. The main objective of these studies was to identify the efficacy of ALS with respect to CRBSI risk rates, catheter exchange rates and related adverse effects.

Conclusions: Antimicrobial Locking Therapy appears significantly beneficial forHD patients as it reduces CRBSI risk and prolongs catheter survival at considerable rates, so it should be seriously considered for systematic useagainst catheter-related infections. Further researchis required to establish safe and effective prevention and therapeutic protocols as alternatives to catheter removal practices.

Keywords: antimicrobial lock therapy, bacteremia, catheter, hemodialysis, catheter removal

\section{Introduction}

The appropriate maintenance of central venous catheters (CVC) is integral for the efficacy and safety of hemodialysiswhich requires systematic catheterization for bloodstream access (Dang et al., 2019). Catheter thrombosis or catheter-related bloodstream infections (CRBSI) are common complications for patients undergoing hemodialysis (Sofroniadou et al., 2017). The probability of blood stream infection (bacteremia) when using dialysis catheters increases exponentially as it reaches significant percentages in only a few weeks (Böhlke et al., 2015). Bacteremia may lead to the rapid deterioration of patients' health as it causes significant clinical signs such as hemodynamic instability, fever, nausea / vomiting and mental disorder (Miller et al., 2016).Consequently, bacteremia increases morbidity, hospitalization frequency, medical cost and mortality (Leistner et al., 2014). Therefore, research in the field of prevention and treatment of CRBSI is imperative for the health management of HD patients.

One of the contemporary approaches for the management of thrombosis and CRBSI inHDpatients include 
Antimicrobial Lock Solutions (ALS) containing anticoagulants and antimicrobial agents (Arechabala, 2018; Labriola, 2017). Although sealing catheters with anticoagulants, such as heparin, is standard for thrombosis prevention, the efficacy of ALS concerning their potential to reduce CRBSI risk requires further investigation. Antibiotics commonly used in ALS are gentamicin, tobramycin, minocycline, cefotaxime, vancomycin and cefazolin, with gentamicin being one of the most extensively studied (Cooney et al., 20014; Golestaneh \& Mokrzycki, 2018). Non-antibiotic agents commonly used in ALS are sodium citrate, taurolidine, EDTA and ethanol (Arechabala, 2018; Dang et al., 2019). Prophylactic use of ALS combining anticoagulants with anti-microbials (antibiotic or non-antibiotic) has been associated with a significant reduction in CRBSI risk by 60\%-70\% (Böhlke et al., 2015; Sun et al., 2020). Moreover, ALS approaches present advantages such as reduced mortality, prevention of biofilm formation and broad range of antimicrobial activity, without risking catheter dysfunction or creation of resistant microbial strains (Raad et al., 2016). The objective of this study was to investigate thesafety and efficacy of the variousALS in the scope to explore the possibilities to endorse the systematic use of ALS for CRBSI prevention and treatment. To this end, this systematic review aims to assess the international literature on antimicrobial lock treatment of dialysis catheters based on data collected throughclinical studies. The assessment includes the identification of the various antimicrobial agents used in locking solutions and the various protocolsfor ALS treatments.

\section{Method}

The search for international peer-reviewed publications for this systematic review was performed through the "Pubmed", "Google Scholar" and "Elsevier" digital databases. The objective of the search was to identify prospective or retrospective clinical studies, randomized clinical trials or cohort studies that involved the prevention or treatment of catheter-related infections in HD patients. The search involved the identification of the key words 'catheter', 'catheter removal', 'hemodialysis' 'bacteremia', 'antimicrobial locktherapy' in the titles of articles in any combination. The search parameters were set for articles from 2015 in the English language. This initial search resulted in $\mathrm{n}=1264$ articles which were subsequently assessed with respect to specific exclusion criteria. According to these criteria $n=815$ articles were excluded as they did not comprise type of studies according to the criteria of the review. The excluded articles were mainly literature reviews, in-vitro studies or did not involve the application of ALS. The references of the excluded articles were further investigated although no additional eligible articles were identified. The remaining $n=449$ articles were further assessed in terms of relevancereading first through the titles, then the abstracts and finally the whole articles excluding $n=218, n=168$ and $n=46$ articles respectively. The majority of articles that were not considered eligible were incomplete in terms of data or involvednon-hemodialysis patients. The articles which were finally considered eligible for this review were $n=17$ (Figure 1). 


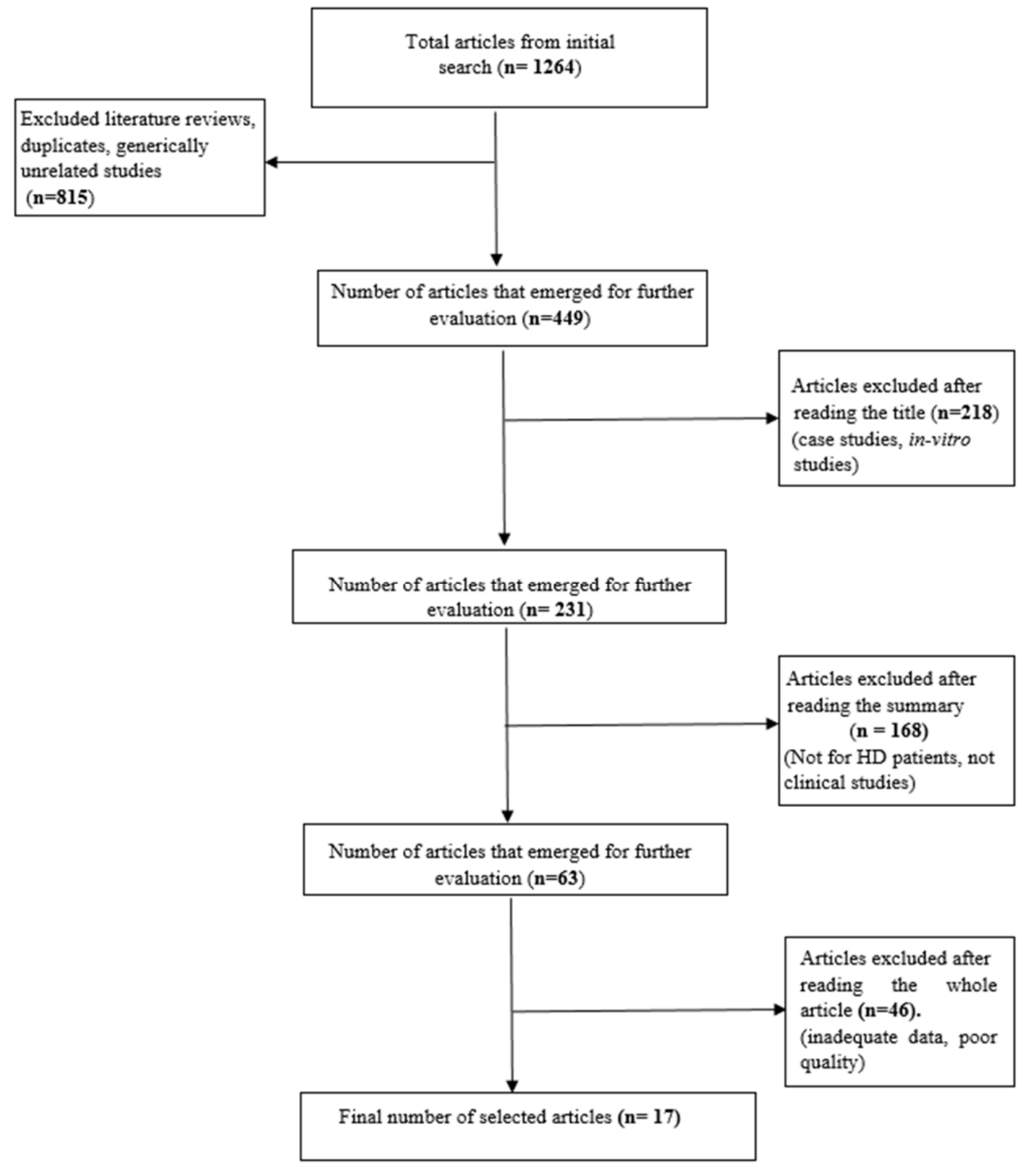

Figure 1. Article selection process

\section{Results}

The selected articles included 4 systematic reviews and 13 prospective or retrospective studies and clinical trials. The articles, their type of study, their objectives and their results are presented in Table 1. 
Table 1. Type, objective and results of selected articles

\begin{tabular}{|c|c|c|c|}
\hline Publication & Type of study & Study objective & Study results \\
\hline \multicolumn{4}{|c|}{ Systematic Reviews } \\
\hline $\begin{array}{l}\text { Zhao et al., } \\
2014\end{array}$ & $\begin{array}{l}\text { Systematic review, } \\
\text { meta-analysis }\end{array}$ & $\begin{array}{l}\text { Evaluation of citrate lock solutions comparing to heparin 'lock' for } \\
\text { the prevention of catheter-related infections and CVC catheter } \\
\text { patency in HD patients. }\end{array}$ & $\begin{array}{l}\text { Catheters with citrate lock solutions containing antimicrobial agents } \\
\text { (citrate }+ \text { gentamicin, citrate }+ \text { taurolidine, and citrate }+ \text { methylene blue }+ \\
\text { methylparaben }+ \text { propylparaben) were more effective at the prevention of } \\
\text { infections than heparin locks at a significant rate }(\mathrm{P}<0.001, \mathrm{P}=0.003 \text {, and } \\
\mathrm{P}=0.008 \text {, respectively). Reduced bleeding episodes with citrate 'locking'. }\end{array}$ \\
\hline $\begin{array}{l}\text { Böhlke et al., } \\
2015\end{array}$ & $\begin{array}{l}\text { Systematic review, } \\
\text { meta-analysis. }\end{array}$ & $\begin{array}{l}\text { Investigation of prevention and surveillance protocols, diagnostic } \\
\text { criteria and management of catheter-related infections in HD } \\
\text { patients. }\end{array}$ & $\begin{array}{l}\text { Gentamicin/citrate or taurolidine/citrate reduced infection rates } \\
\text { substantially. The review concludes that the most effective approach to } \\
\text { treat catheter-related infections in HD patients is still catheter removal. } \\
\text { Catheters may be salvaged using antibiotic lock and systemic } \\
\text { antibiotics.Antibiotic lock as prophylactic measure may reduce infection } \\
\text { rates or severity. Further research required. }\end{array}$ \\
\hline $\begin{array}{l}\text { Zhao et al., } \\
2018\end{array}$ & $\begin{array}{l}\text { Systematic review, } \\
\text { meta-analysis }\end{array}$ & $\begin{array}{l}\text { Evaluation of the effect of ethanol lock comparing to other } \\
\text { 'locking' solutions, on catheter-related bacteremia in HD patients } \\
\text { with central venous catheters. }\end{array}$ & $\begin{array}{l}\text { Ethanol lockingwas found effective in reducing the risk of CRBSI at a } \\
\text { significant rate with RR } 0.54(95 \% \text { CI } 0.38-0.78 ; \mathrm{I} 2=0 \% ; \mathrm{p}=0.001)\end{array}$ \\
\hline Mai et al., 2019 & $\begin{array}{l}\text { Systematic review, } \\
\text { meta-analysis }\end{array}$ & $\begin{array}{l}\text { Evaluation of citrate lock solutions comparing to heparin lock for } \\
\text { the prevention of catheter-related complications in HD patients. }\end{array}$ & $\begin{array}{l}\text { Antimicrobial-containing Citrate locks reduce the risk of } \\
\text { bacteremiacomparing to heparin locks (RR: } 0.34,95 \% \mathrm{CI} 0.24-0.49 ; \mathrm{I} 2= \\
0 \% ; \mathrm{P}<0.00001) \text {. Risk of bleeding and exit point infection were also } \\
\text { reduced. }\end{array}$ \\
\hline \multicolumn{4}{|l|}{ Clinical studies } \\
\hline $\begin{array}{l}\text { Kanaa et al., } \\
2015\end{array}$ & $\begin{array}{l}\text { Multicenter, } \\
\text { randomized, } \\
\text { controlled trial. }\end{array}$ & $\begin{array}{l}\text { Assessment of Cathasept } 4 \% \text { locks comparing to heparin } 5,000 \\
\mathrm{U} / \mathrm{mL} \text { locks with respect to catheter microbial colonization of the } \\
\text { catheter, CRBSI rate, catheter patency, and biomarkers of } \\
\text { inflammation and anemia in HD patients. }\end{array}$ & $\begin{array}{l}\text { In } 117 \mathrm{HD} \text { patients from } 4 \mathrm{HD} \text { centers Cathasept locks significantly } \\
\text { reduced catheter microbial colonization }(0.14 / 1,000 \text { catheter-days) } \\
\text { comparing to heparin locks } 1.08 / 1,000 \text { catheter-days. Reduction in CRBSI } \\
\text { rate }(0.28 / 1,000 \text { catheter-days) was not statistically significant comparing } \\
\text { to heparin }(0.68 / 1,000 \text { catheter days) and resulted to more thrombotic } \\
\text { incidents. }\end{array}$ \\
\hline $\begin{array}{l}\text { Moghaddas et } \\
\text { al., } 2015\end{array}$ & $\begin{array}{l}\text { Multicenter, } \\
\text { randomized } \\
\text { controlled trial }\end{array}$ & $\begin{array}{l}\text { HD patients randomized to receive either heparin }(2500 \mathrm{U} / \mathrm{ml}) \text { or a } \\
\text { mixture of } 10 \mathrm{mg} / \mathrm{ml} \text { cotrimoxazole (trimethoprim-based) and } \\
2500 \mathrm{U} / \mathrm{ml} \text { heparin as catheter lock solutions to determine } \\
\text { differences in CRBSI risk ratios and catheter survival period. }\end{array}$ & $\begin{array}{l}\text { In } 87 \mathrm{HD} \text { patients ( } 46 \text { cotrimoxazol group, } 41 \text { Heparin group), CRBSIs } \\
\text { rates per } 1000 \text { catheter-days ware significantly lower }(0.58 \text { vs } 4.4 \text { events; } p \\
=0.002) \text { in the cotrimoxazole group. Infection-free catheter survival } \\
\text { period was significantly longer (log rank statistic } 5.88 ; p=0.015) \text { in the } \\
\text { cotrimoxazole group. No statistical differences regarding thrombosis or } \\
\text { catheter removal rates. }\end{array}$ \\
\hline
\end{tabular}


Assessment of the efficacy of systemic daptomycin (DPT) plus retrospective study

15 HD patients were treated with DPT-ALT for CRBSI $(9$ with coagulase-negative Staphylococcus, 2 with MRSA, 3 with MSSA and 1 with polymicrobial infections). $73.3 \%$ of the patients recovered and catheter removal was avoided. Treatment failed in all MRSA cases.Results were considered particularly promising.

Comparison of Taurolock/Hep and taurolidine citrate with urokinase (Taurolock/U) catheter lock solutions with respect to catheter-related dysfunction episodes including acute catheter thrombosis, requirement of recombinant tissue plasminogen activator (rt-PA), CRBSI incidences, dialysis inadequacy and blood flow rates in HD patients.

Al-Ali et al., randomized, controlled trial.
Barcellos et al. Randomized,

2017 controlled trial
Assessment of the effectiveness of $30 \%$ trisodium citrate (TSC30 \%) locks on subclavian and jugular vein CVC lock in HD patients comparing to heparin locks with respect to CRBSI rates and catheter functionality.

In $85 \mathrm{HD}$ patients in the Taurolock/Hep group and $79 \mathrm{HD}$ patients in the Taurolock/U group, catheter exchange rate due to thrombosis or CRBS was significantly lower in Taurolock/U group $(\mathrm{P}=0.028)$. rt-PA requirement was significantly less in the Taurolock/U group than in the Taurolock/Hep group $(\mathrm{P}=0.006)$

In $464 \mathrm{HD}$ catheters (231 TSC30 \% group, 233 heparin group), the CRBSI-free survival of the TSC30 \% group was significantly shorter. CRBSI-free survival was reduced only for subclavian CVCs Functionality was not found significantly different between groups although survival was shorter for subclavian CVC locked with TSC30\%. Differences may be related to anatomical effects and CVC design rather than locking effects.

103 HD patients were randomized in an ethanol/heparin group (52) and a heparin group (51). The CRBSI incidents for the ethanol/heparin group

Sofroniadou, Prospective, et al., 2017 controlled study.
Investigation of ethanol lock solutions for the prevention of CRBSI in HD patients. Exit site infections, thrombotic and bleeding episodes were also investigated.

\begin{tabular}{lll}
\hline $\begin{array}{l}\text { Winnicki et } \\
\text { al.,2017 }\end{array}$ & $\begin{array}{l}\text { Prospective } \\
\text { multicenter } \\
\text { randomized } \\
\text { controlled trial }\end{array}$ & $\begin{array}{l}\text { Assessment of } 2 \text { lock regimens using } 3 \text { commercial catheter locks } \\
\text { (TauroLock }{ }^{\mathrm{TM}}-\mathrm{Hep} 500, \text { TauroLock } \\
\text { solution). The assessment concerned CRBSI prevention, catheter } \\
\text { functionality and cost. }\end{array}$ \\
\hline $\begin{array}{l}\text { Vercaigne et al., } \\
2017\end{array}$ & $\begin{array}{l}\text { Prospective, } \\
\text { randomized, } \\
\text { study }\end{array}$ & $\begin{array}{l}\text { Assessment of } 30 \% \text { ethanol/4\% sodium citrate and heparin } 1000 \\
\text { units/mL locking solutions to identify and compare adverse } \\
\text { effects, risk for CRBS and catheter functionality in HD patients. } \\
\text { Ultimate objective to avoid catheter removal. }\end{array}$
\end{tabular}
was $7.69 \%$ while for the heparin group it was $21.57 \%(\mathrm{P}=0.04)$. CRBSI rates per 1000 catheter days were 2.53 and 6.7 respectively $(\mathrm{P}=0.04)$. Ethanol locking reduced CRBSI rates although the mean cumulative infection-free catheter survival did not differ significantly (log-rank test $=2.99, \mathrm{P}=0.08$ ). Frequency of thrombotic episodes did not differ.

In $106 \mathrm{HD}$ patients, 6 CRBSI incidents occurred in 6 of 52 patients in the taurolidine group and 18 incidents occurred in 13 of 54 patients in the citrate group, corresponding to 0.67 and 2.7 episodes of CRBSI per 1000 catheter days.Taurolidine-based lock solutions + heparin/urokinase significantly reduced CRBSI risk comparing to $4 \%$ citrate solutions.

In $40 \mathrm{HD}$ patientsrandomized to $30 \%$ ethanol $/ 4 \%$ sodium citrate or heparin 1000 units $/ \mathrm{mL}$ locking solution, catheter survival was longer in the ethanol/citrate group (2.8/1000 catheter days in the ethanol/citrate group, $1.5 / 1000$ catheter days in the heparin group). Ethanol/sodium citrate locking appears more efficient in reducing CRBSI risk and avoiding catheter removal comparing to heparin locks. 


$\begin{array}{lll}\text { Rijnders et al., } & \begin{array}{l}\text { Randomized, } \\ \text { evaluator-blinded } \\ \text { clinical trial }\end{array} & \begin{array}{l}\text { Assessment of the efficacy of trimethoprim } 5 \mathrm{mg} / \mathrm{mL} \text {, ethanol } \\ 25 \% \text {, and Ca-EDTA 3\% (IMD) lock solution comparing to heparin }\end{array} \\ & 5000 \mathrm{U} / \mathrm{mL} \text { for the prevention of CRBSI in HD patients. }\end{array}$

Rijnders et al., evaluator-blinded
2018 $5000 \mathrm{U} / \mathrm{mL}$ for the prevention of CRBSI in HD patients.
In $270 \mathrm{HD}$ patients monitored for 43738 CVC-days, despite the low CRBSI rate in both groups $(0.41 / 1000 \mathrm{CVC}$-days $)$, the rate in the IMD group was significantly lower by 4.56 -fold to $0.09 / 1000$ CVC-days (P $<.03$ ). Comparable adverse effects and rate of catheter removal.

113 cases of CRBSI were identified while monitoring 274 catheters in HD patients who receivedsystemic and lock therapy with Cephazolin and/or

Mandolfo et Monocentric To instigate the possibility to avoid catheter removal in HD 2019 randomized clinical patients with S.aureus CRBSI using systemic and antibiotic 'lock' study therapy. CRBSI, 34 were successfully treated (85\%) avoiding catheter removal. In 19 episodes of MRSA and 21 of MSSA, 79\% and 90\% were successfully treated respectively. Recurrences were observed at $22 \%$ of the cases, $75 \%$ of which were treated successfully.

Prospective Comparing the efficacy gentamicin In 65 HD patients in the gentamicin/cafazolin group and $62 \mathrm{HD}$ patients in

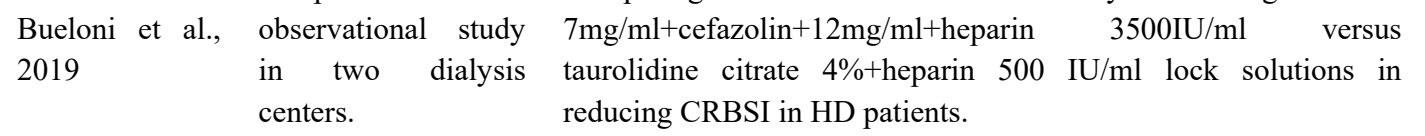
the two groups concerning CRBSI $(\mathrm{p}=0.18)$ or exit site infection rates $(\mathrm{p}=0.37)$.

CRBSI rates were measured before and after and intervention of prolonged

Padilla-Orozco Prospective cohort Evaluation of the utility of a generalized and prolonged et al., 2019 study gentamicin-lock therapy in HD patients with respect to the reduction of CRBSI rates.

gentamicin lock therapy. CRBSI incidents were reduced significantly from 1.28 to 0.2 cases per 1,000 catheter-days $(\mathrm{p}=0.001)$ especially with $P$. aeruginosa and other Gram-negative bacteria. Prolonged gentamicin was considered safe and effective.

Assessment of urokinase plasminogen activator (uPA)-based (+/antibiotics) comparing to heparin-based lock solutions on HD patient catheters with respect to CRBSI rates and adverse effects and in-vitro study involving the induction of biofilm formation in HD catheters to mimic CRBSI development. uPA +/- antibiotics was applied on the induced biofilms to study its antimicrobial and biofilm removing properties.
In $86 \mathrm{HD}$ patients (34 heparin-based locks, 52 uPA-based locks +/antibiotics), uPA-based lock solutions were found more effective in treating coagulase-negative Staphylococci related CRBSI than other microorganisms without significant adverse effects. uPA+antibiotics presented better antimicrobial and antibiofilm properties than uPA alone or heparin-based lock solutions both in vitro and in vivo. 


\subsection{ALS Consistency}

The ALS used in these studies contained various combinations of antibiotic and non-antibiotic agents. Concerning the non-antibiotic agents, heparin-based lock solutions were widely used throughout these studies, as heparin is systematically used to prevent catheter thrombosis and in many studies it was used in the control groups (Zhao et al., 2014; Zhao etal., 2018; Mai et al., 2019; Kanaa et al., 2015; Moghaddas et al., 2015; Rijnderset al., 2018; Wang et al., 2020).

Citrate-based lock solutions were also extensively studied (Mai et al., 2019; Winnicki et al., 2017; Barcellos et al., 2017). Citrate is a non-antibiotic agent often used in catheter lock solutions as an alternative to heparin due to its anticoagulant activity (Bruyère et al., 2014). Citrate was investigated in various combinations with antibiotics and other non-antibiotic agents such as Citrate/Gentamicin (Zhao et al., 2014), Citrate/Taurolidine +/- heparin (Zhao et al., 2014; Al-Ali et al., 2017; Winnickiet al., 2017; Bueloni et al., 2019), Citrate/Taurolidine/urokinase (Al-Ali et al., 2017; Winnicki et al.,2017) and Citrate/methylene blue/methylparaben/propylparaben (Zhao et al., 2014). Taurolidine, which is a synthetic non-antibiotic agent with broad antimicrobial and anticoagulant activity (Torres-Viera et al., 2000), is regularly used in catheter locks and it is commercially available in combination with citrate and heparin (Taurolock/Hep) or urokinase (Taurolock/U). These commercially available lock solutions with taurolidine were used in the studies by Al-Ali et al., 2017 and Winnickiet al., 2017 in various combinations and concentrations. Urokinase plasminogen activator (uPA) was investigated as a non-antibiotic agent in locking solutions for its thrombolytic properties in combination with antibiotics (Bonkain et al., 2017). Ethanol was also studied as an ALS component due to its antimicrobial properties in various concentrations and combinations (Sofroniadou, et al., 2017; Zhao et al., 2018). Cathasept (4\% tetra-sodium EDTA) was also studied byKanaa et al., 2015 for its antimicrobial/anticoagulant properties.

Concerning the antibiotics used in the studies included in this review, they were investigated either as single components or in combinations +/- anticoagulants in ALS such as Cefazolin/Gentamicin/heparin (Bueloni et al., 2019), Cefazolin/Vancomycin (Mandolfo et al.,2019), Cotrimoxazole (Moghaddas et al., 2015), Daptomycin (Yenet al., 2015), Trimethoprim/ethanol/Ca-EDTA 3\% (IMD) (Rijnderset al., 2018), Gentamicin (Padilla-Orozco et al., 2019).

\subsection{Objectives of the Studies}

Concerning the main objective of the studies, the majority aimed at the investigation of the efficacy of lock solutions to prevent or treat catheter related infections in HD patients in the scope to prolong catheter survival and avoid catheter exchange. More specifically, all the meta-analyses (Zhao et al., 2014; Böhlke et al., 2015; Zhao et al., 2018; Mai et al., 2019) aimed to investigate the effect of various lock solutions on the CRBSI risk patients with the ultimate objective to identify potential prevention/therapeutic protocols (Böhlke et al., 2015). The investigation was either aiming to compare specific non-antibiotic lock solutions (Zhao et al., 2014; Zhao et al., 2018; Mai et al., 2019) against heparin-based controls or to compare the most commonly used antibiotic/non-antibiotic ALS against each other in various combinations (Böhlke et al., 2015). Other relevant objectives which were common among these meta-analyses wereto assess catheter flow rate and dialysis efficiency, exit-site infection, adverse effects such as catheter-relatedthrombosis, bleeding and clinical side-effects, rate of thrombolytic treatment requirement (Zhao et al., 2014), catheter types and insertion sites (Böhlke et al., 2015; Barcellos et al., 2017; Zhao et al., 2018), catheter CRBSI-free survival, catheter duration and all-cause mortality (Zhao et al., 2014; Mai et al., 2019). The meta-analyses also assessed the heterogeneity, the sample size and the publication bias of the studies they included.The aforementionedobjectives were also common among most of the clinical studies included in this review. Secondary objectives which were distinctive in specific clinical studies werethe investigation of biomarkers for inflammation and anemia (Kanaa et al., 2015), the CRBSI risk assessment for specific microorganism (Yen et al., 2015; Mandolfo et al., 2019; Wang et al., 2020), the cost-effectiveness of specific treatments (Winnicki et al., 2017), differences with respect to catheter insertion sites (Barcellos et al., 2017) and the anti-biofilm properties of antimicrobial/anticoagulant agents in certain ALS treatments (Wang et al., 2020).

\subsection{Study Results}

The 4 meta-analyses in this study concerning the efficacy of ALS for the prevention or treatment of CRBSI in HD patients agree thatlocking therapy appears to have significant potential benefits. The reviews concluded that overall ALS therapy reduces CRBSI risk and prolongs catheter survival without profound adverse effects on the patients' health or dialysis efficiency. More specifically, citrate-based ALS +/- antibiotics were found to significantly reduce the risk for CRBSI comparing to heparin locks, without increasing bleeding or catheter thrombosis incidents (Zhao et al., 2014; Böhlke et al., 2015; Mai et al., 2019). Ethanol locking was also found to reduce the risk of bacteremia in HD patients at a significant rates (Zhao et al., 2018). All reviews though emphasize 
that in order to endorse the systematic use of ALS against CRBSI, further research is required as no prevention protocols and diagnostic criteria are yet established. Furthermore, concerning the use of ALS as an effective alternative to avoid catheter removal, current research is not yet conclusive. Böhlke et al. (2015) in particular claim in their review that catheter removal was found to be the most effective approach to treat CRBSI while certain adverse effects that might be related to systematic ALS treatment, such as hypocalcaemia or aminoglycoside ototoxicity, should be seriously taken into account.

The results of the clinical studies in this review are generally in agreement with the results of the systematic reviews. A considerable percentage of the clinical studies that investigated the effect of various ALS $+/$ - antibiotics comparing to heparin locks found that ALS reduced CRBSI risk at a significant rate without major adverse effects (Moghaddas et al., 2015; Rijnders et al., 2018; Padilla-Orozco et al., 2019; Wang et al., 2020) with the exception of the study of Kanaa et al. (2015) where Cathasept locking did not result at a significant reduction of CRBSI rate comparing to heparin although bacterial colonization was significantly reduced. Similarly, Sofroniadou et al. (2017) detected reduced CRBSI incidents with ethanol/heparin locks comparing to heparin control at non-significant rates. Moreover, Vercaigne et al. (2017) although identified reduced CRBSI risk using 30\% ethanol $/ 4 \%$ sodium citrate locks, the significance of their results cannot be determined as this was a small scale pilot study. It's worth mentioning that Barcellos et al. (2017) were the only to report reduced CRBSI-free catheter survival with locking therapy (citrate-based lock) comparing to heparin control although they conclude that their results might have been affected by anatomical andCVC design differences related to catheter insertion site. More specifically they report that reduced CRBSI-free catheter survival was detected only with subclavian catheters while no significant differences were detected with jugular catheters. Concerning the studies that aimed to investigate the use of ALS to avoid catheter removal, Yen et al. (2015) consider Daptomycin lock to be particularly promising similarly to Vercaigne et al. (2017) who also identified increased possibilities to avoid catheter removal with ethanol/citrate. Both studies though, are small scale pilot studies and cannot provide statistically significant data. In a similar context, Mandolfo et al. (2019) claim that catheterrescue percentages may be much higher than presented in contemporary literature through systemic and antibiotic lock therapyand stress that prompt detection and treatment initiationare critical for catheter rescue. Concerning the comparison of the efficacy of specific lock solutions, Al-Ali et al. (2017) and Winnicki et al. (2017) focus on the investigation of TauroLock ${ }^{\mathrm{TM}}$-Hep (taurolidin/heparin) and TauroLock ${ }^{\mathrm{TM}}$-U (taurolidin/urokinase) which are commercially available taurolidine-based lock solutions.Al-Ali et al. (2017) concluded that TauroLock ${ }^{\mathrm{TM}}-\mathrm{U}$ reduces total number catheter exchange due to thrombosis or CRBSI at a significant rate comparing to TauroLock ${ }^{\mathrm{TM}}$-Hep while Winnicki et al. claim that Taurolidine-based lock solutionssignificantly reduced CRBSI risk comparing to citrate-based solutions. On the other hand, Bueloni et al. did not detect significant differences in CRBSI or catheter exchange rates when comparing taurolidine/citrate locks with antibiotic (gentamicin/cafazolin)based locks (Bueloni et al., 2019). Regarding the effect of urokinase, Wang et al. (2020) concluded that urokinase plasminogen activator (uPA) with antibiotics presented better antimicrobial and antibiofilm properties than uPA alone or heparin-based locks both in vitro and in vivo. What is also worth mentioning concerning the effect of ALS on specific microorganism, MRSA infections appear more challenging to eradicate comparing to MSSA and coagulase-negative Staphylococcus, although these results are preliminary and require further research (Yen et al., 2015; Mandolfo et al., 2019). Moreover, Padilla-Orozco et al. (2019) documented that gentamicin locks are the most effective on P. aeruginosa and other Gram-negative bacteria.

\section{Discussion}

Currently, the CDC recommends ALS treatment only for HD patients with recurrent bloodstream infections as there are no conclusive data on the efficacy and safety of ALS for long-term preventative or therapeutic protocols. This review was designed in the view to investigate the prospects of recommending the systematic application of ALS against CRBSI for HD patients as an alternative to catheter removal. Catheter removal and exchange is a common practice for the treatment ofCRBSI (Böhlke et al., 2015) which can be quite challenging for patients on regular catheterizations such as HD patients, cancer patients or patients on parenteral nutrition (Hachem et al., 2018). ALS treatment is considered promising for systematic application as it appears to have not only significant effect on CRBSI rates (Sun et al., 2020) but also presents other considerable advantages such as cost-effectiveness (Leistner et al., 2014;Winnicki et al.,2017) and anti-biofilm properties. The microbial biofilm that is formed in the lumen of infected catheters is often resistant to systemic antibiotics, so biofilm prevention is essential for CRBSI treatment (Pérez-Granda et al., 2017; Wang et al., 2020). Moreover, antimicrobial agents in locking solution maintain prolonged, high concentration within the catheter lumen with minimal systemic absorption (Bookstaver et al., 2009) and therefore have not been associated with increased risk for the formation of resistant microbial strains (Raad et al., 2016). Apparently, the evaluation of the prospects for systematic use of ALS is imperative as it 
may contribute significantly to the wellbeing of HD patients as well as all patients undergoing regular catheterizations.

Regarding the variouslock solutions currently investigated in this field of research and more specifically the commonly used non-antibiotic ALS components, citrate-based ALS are among the most extensively studied (Mai et al., 2019). According to the meta-analysis of Mai et al., citrate-based locking including antimicrobials reduces significantly CRBSI risk in HD patients comparing to conventional heparin locks without affecting the risk of catheter removal, the frequency of thrombolytic treatment, thrombocytopenia rates or all-cause mortality (Mai et al., 2019). This is consistent with the review of Zhao et al. where citrate + antimicrobials is found to reduce CRBSI risk in HD catheters but not citrate alone (Zhao et al., 2014). On the other hand Liu et al. found significant CRBSI reduction with citrate locks even without antimicrobials (Liu et al., 2015). Vercaigne et al. also report that citrate is potentially more effective than heparin in CRBSI prevention in their pilot study for HD patients (Vercaigne et al., 2017). Mai et al. claim that such inconsistencies are due to the fact that previous studies did not systematically considercritical parameters that may affect citrate efficacy such as catheter type or type of infection. For example, Mai et al. found that CRBIS risk is reduced with tunneled cuffed catheterscomparing to non-tunneledones while CRBSI occurrence is more closely related to S.aureus with citrate than heparin locking.Abe et al. also reported no significant difference to CRBSI rates with citrate comparing to heparin locks with non-tunneled catheters which are primarily used in Egypt for dialysis (Abe et al., 2018). Taking into account parameters such as type of catheter or infection in their review Mai et al., concluded that citrate alone does not seem to reduce CRBSI risk while further research is required for the establishment of specific locking protocols (Mai et al., 2019). Similarly, Barcellos et al. in their clinical trial on the effectiveness of citrate locks comparing to heparin concluded that their results were inconclusive as the insertion site of the catheter in HD patients seemed to affect significantly the CRBSI-free catheter survival. More specifically, CRBSI-free catheter survival did not differ significantly between citrate (-antimicrobials) and heparin locks with jugular vein catheters, which is consistent with the results of Zhao et al. and Mai et al., while with subclavian catheters CRBSI-free survival was significantly lower with citrate locks (Barcellos et al., 2017). Other studies on the efficacy of citrate locks against CRBSI highlight on the significance of asepticcatheter manipulation in accordance to CDC guidelines, as imperfect sterility may compromise locking efficiency (Cooney et al., 2020). To conclude, citrate may be an effective anticoagulant alternative to heparin and contribute to CRBSI risk reduction in combination with antimicrobials but further research is required for the standardization of detailed protocols (May et al., 2019).

Ethanol is another promising non-antibiotic catheter lock that is easily available, inexpensive, with wide antimicrobial properties that successfully penetrates microbial biofilm and does not facilitate the development of resistant microbial strains as opposed to antibiotic locks (Balestrino et al., 2009). Ethanol locking therapy for the prevention of bacteremia is associated with encouraging results in contemporary research. Sofroniadou et al. in their clinical study detected reduced CRBSI risk with ethanol/heparin locking comparing to heparin control although the mean cumulative infection-free catheter survivalwas not significantly different in the two groups (Sofroniadou et al., 2017). Zhao et al. report that ethanol locking decreases significantly the risk for CRBSIfor HD patients without significant adverse effects, although this result concerns only tunneled catheters (Zhao et al., 2018). Tunneled catheters are believed to offer better antimicrobial protection comparing to un-tunneled ones, so it is possible that ethanol cannot prevent bloodstream infections unless the major cause for CRBSI, which is the migration of microorganism from the skin to the bloodstream along the catheter, is eliminated (Timsit et al., 1999). This meta-analysis also points out that locking effect appears more beneficial with patients in higher risk for CRBSI, such as patients with hematological malignancy, while the protective effect of locking therapy is not easily measurable in patients with milder health conditions. Apparently, subgroup analysis is imperative for the accurate evaluation of the effectiveness of ethanol-based locking against CRBSI in HD patients as flawed research methodology may be masking results (Zhao et al., 2018).

Taurolidineis another antimicrobial agent that is commonly used in ALS due to its antibiofilm and antimicrobial properties against Gram-positive and Gram-negative bacteria including MRSE, CoNS and vancomycin-resistant enterococci, while it has not been associated with serious side effects or bacterial resistance (Torres-Viera et al., 2000). Liu et al. in their meta-analysis estimated a $66 \%$ reduction in CRBSI risk with taurolidine locks while Zwiechet al. reported a 77\% decrease in their review (Liu et al., 2013; Zwiech et al., 2016). Norris et al. though, concluded that taurolidine is potentially beneficial against bloodstream infections in high risk cancer patients but further research is required for conclusive results (Norris et al., 2017). The more recent meta-analysis of Sun et al., for CRBSI risk in pediatric patients, reportsa significant reduction of CRBSI incidences with taurolidine locks but indicates that thequality of the studies included in their review was poor (Sun et al., 2020). No studies on taurolidine as a single antimicrobial agent in lock solutions specifically for HD patients were identified. Overall, 
the exact contribution of taurolidine as a single antimicrobial agents in locking solution against CRBSI is yet to be determined as large scale high-quality clinical trials are required (Sun et al., 2019).

Considerable research has also been done on the commercially available TauroLock ${ }^{\mathrm{TM}_{-}}$Hep (taurolidine/heparin) and TauroLock ${ }^{\mathrm{TM}}-\mathrm{U}$ (taurolidine/citrate/urokinase)(Al-Ali et al., 2017; Winnicki et al., 2017). These studies though, focused primarily on the antithrombotic effect of urokinase in theTauroLock ${ }^{\mathrm{TM}}-\mathrm{U}$. Urokinase plasminogen activator (uPA) is a fibrinolytic protease with thrombolytic properties which has been associated with reduced CRBSI incidents and improved catheter functionality (Bonkain et al., 2017). Both Al-Ali et al. and Winnichi et al. report reduced rates of all-causes catheter exchange concluding that Taurolock/ $\mathrm{U}$ is a safe and effective lock solution for tunneled dialysis catheters (Al-Ali et al., 2017; Winnicki et al.,2017). These results were also corroborated by the studies of Bonkain et al. and Van Hulle et al. who report that urokinase locks are significantly efficient in preventing thrombotic dysfunction or restoring catheter functionality (Bonkain et al., 2017; Van Hulle et al., 2019). Finally, Wang et al. in their combined in vivo and in vitro study on hemodialysis catheter locks demonstrated that uPA locks have superior anti-thrombotic and anti-biofilm properties comparing to heparin locks while uPA combined with antibiotics also offers enhanced antimicrobial protection (Wang et al., 2020).

Cathasept (4\% tetra-sodium EDTA) has also been studied for its anticoagulant and antimicrobial properties as a lock solution component (Kanaa et al., 2015; Hill et al., 2020). Cathasept has been reported to reduce CRBSI rates significantly in parenteral nutrition patients (Hill et al., 2020). Kanaa et al. in their clinical trial on HD patients, though they had detected significantly reduced microbial colonization in HD catheters with cathasept, they could not confirm a significant reduction in CRBSI rates comparing to heparin so they stressed the need for further research based on higher quality methodology (Kanaa et al., 2015). Quirt et al. in a more recent study on pediatric intestinal failure patients reported that according to their preliminary findings $4 \%$ tetrasodium EDTA solution effectivelyreduced catheter-related bloodstream infections and catheter flow (Quirt et al. 2021). EDTA (ethylenediaminetetraacetic acid) as the main component of cathasept and a chelating agent with known antimicrobial/anticoagulant/antibiofilm and blood vessel protective activity (Song \& Zhang, 2020) has been extensively studied as a lock solution candidate (Hachem et al., 2018; Dang et al., 2019). More specifically, Dang et al. in their meta-analysis estimated that EDTA is the most effective against CRBSI and exit-site infection among 13 different lock solutions at a rate of $84.4 \%$. EDTA has also been reported to complement successfully the antimicrobial activity of antibiotic-based locks (Raad et al., 2016; Rijnders et al., 2018). Although EDTA has been extensively studied as a catheter lock component, further research and subgroup analysis for the establishment of specific protocols is yet required (Dang et al. 2019).

Antibiotic lock solutions exhibit broad antimicrobial activity, reduce CRBSI and patients' mortality. The disadvantages of prolonged antibiotic locking though,include difficulty to infiltrate microbial biofilm in catheter lumen,increased risk for development of resistant microbial strains, systemic toxicity and catheter dysfunction (Golestaneh \& Mokrzycki, 2018). Gentamicin has been reported to significantly reduce CRBSI risk and mortality hazard comparing to non-antibiotic locks in HD patients (Moore et al., 2014). Padilla-Orozco et al. concluded that gentamicin locks are significantly effective against hemodialysis catheter infections, especially with $\mathrm{P}$. aeruginosa and other Gram-negative bacteria. Bolke et al. also identify the effectiveness of gentamicin-based locks against bloodstream infections in HD patients but raise concerns about the measurable levels of gentamicin in patients' plasma due to systemic absorption, which indicates a risk for development of gentamicin-resistant microbes (Böhlke et al., 2015). Concerning the studies comparing antibiotic-based to non-antibiotic locks, Moghaddas et al. (2015), Mandolfo et al. (2019) and Yen et al. (2015) identified significant or quite promising advantages with antibiotic locks using cotrimoxazol, daptomycin and caphazolin/vancomycin respectively. More specifically, Mandolfo et al. (2019) support that systematic antibiotic locking therapy should be seriously considered as a prophylactic practice for HD patients to reduce catheter removal rates. This view is corroborated by the review of Yahav et al. (2008) who concluded that antibiotic catheter locks should be considered as a routine clinical practice.On the other hand, Rijnders et al. (2018) and Bueloni et al. (2019) in their studies with trimethoprimand gentamicin/cefazolin respectively did not detect measurable differencescomparing to non-antibiotic locks. These inconsistencies highlight the need for further research based on subgroup analysis and high quality clinical trials as most researchers point out in recent relevant reviews (Böhlke et al., 2015; Dang et al., 2019; Sun et al., 2020).

\section{Conclusion}

The studies presented and analyzed in this review demonstrate encouraging emerging data on the application of antimicrobial lock solutions that could potentially be used systematically to reduce or treat CRBSI, prolong catheter survival and decrease the necessity for catheter removal in HD patients. These data although promising, are not yet conclusive and cannot fully support the endorsement of the systematic use of lock solutions to avoid 
catheter removal, which is the primary response to catheter-related bloodstream infections. A significant number of researchers in this field of study identify the need for further research based on large scale, high quality clinical trials. More specifically, they emphasize on the heterogeneity among studies, the shortage of large scale trials and the likelihood for publication bias. The necessity for subgroup analysis is also emphasized as results may be biased in populations in higher risk for bloodstream infection. Type of catheter, type of microbial infection, catheter handling and catheter insertion site may also comprise independent parameters that should be considered in subgroup analysis as they may mask results. Overall, locking therapy appears to be a highly advantageous tool in the battle against thedetrimental effects of catheter related infections, so further research on the prospects of the various lock solutions is imperative as it might lead to a significant improvement in the wellbeing of HD patients.

\section{Acknowledgements}

I would like to thank Dr. M.Tzanakakis for his guidance and support throughout this study. I would also like to thank H. Aslanoglou for her indispensable help with the editing of this paper.

\section{Competing Interests Statement}

The authors declare that there are no competing or potential conflicts of interest.

\section{References}

ABE, A. A., ElSaid, T. W., El Said, H. W., Hemida, W., Zaghlool, S., Ramadan, A., ... \& Ali, H. M. (2018). A randomized controlled clinical trial of $4 \%$ sodium citrate versus heparin as locking solution for temporary dialysis catheters among hemodialysis patients. Clinical nephrology, 90(5), 341-349. https://doi.org/10.5414/CN109162

Al-Ali, F., Hamdy, A. F., Hamad, A., Elsayed, M., Zafar Iqbal, Z., Elsayed, A., ... \& Fawzy, A. (2018). Safety and efficacy of taurolidine/urokinase versus taurolidine/heparin as a tunneled catheter lock solution in hemodialysis patients: a prospective, randomized, controlled study. Nephrology Dialysis Transplantation, 33(4), 619-626. https://doi.org/10.1093/ndt/gfx 187

Arechabala, M. C., Catoni, M. I., Claro, J. C., Rojas, N. P., Rubio, M. E., Calvo, M. A., \& Letelier, L. M. (2018). Antimicrobial lock solutions for preventing catheter-related infections in haemodialysis. Cochrane Database of Systematic Reviews, (4). https://doi.org/10.1002/14651858.CD010597.pub2

Barcellos, F. C., Nunes, B. P., Valle, L. J., Lopes, T., Orlando, B., Scherer, C., ... \& Böhlke, M. (2017). Comparative effectiveness of $30 \%$ trisodium citrate and heparin lock solution in preventing infection and dysfunction of hemodialysis catheters: a randomized controlled trial (CITRIM trial). Infection, 45(2), 139-145. https://doi.org/10.1007/s15010-016-0929-4

Böhlke, M., Uliano, G., \& Barcellos, F. C. (2015). Hemodialysis catheter-related infection: prophylaxis, diagnosis and treatment. The journal of vascular access, 16(5), 347-355. https://doi.org/10.5301/jva.5000368

Balestrino, D., Souweine, B., Charbonnel, N., Lautrette, A., Aumeran, C., Traoré, O., \& Forestier, C. (2009). Eradication of microorganisms embedded in biofilm by an ethanol-based catheter lock solution. Nephrology Dialysis Transplantation, 24(10), 3204-3209. https://doi.org/10.1093/ndt/gfp187

Bonkain, F., Van Hulle, F., Janssens, P., Catalano, C., Allamani, M., Stolear, J. C., ... \& Wissing, K. M. (2017). Urokinase-containing locking solution in the prevention of dialysis catheter dysfunction: a double blind randomized controlled trial. The journal of vascular access, 18(5), 436-442. https://doi.org/10.5301/jva.5000737

Bookstaver, P. B., Williamson, J. C., Tucker, B. K., Raad, I. I., \& Sherertz, R. J. (2009). Activity of novel antibiotic lock solutions in a model against isolates of catheter-related bloodstream infections. Annals of Pharmacotherapy, 43(2), 210-219.https://doi.org/10.1345/aph.1L145

Bruyère, R., Soudry-Faure, A., Capellier, G., Binquet, C., Nadji, A., Torner, S., ... \& Quenot, J. P. (2014). Comparison of heparin to citrate as a catheter locking solution for non-tunneled central venous hemodialysis catheters in patients requiring renal replacement therapy for acute renal failure (VERROU-REA study): study protocol for a randomized controlled trial. Trials, 15(1), 1-8. https://doi.org/10.1186/1745-6215-15-449

Bueloni, T. N. V., Marchi, D., Caetano, C., de Souza Cavalcante, R., Amaral, M. L. M., \& Ponce, D. (2019). Cefazolin-gentamicin versus taurolidine-citrate for the prevention of infection in tunneled central catheters in hemodialysis patients: A quasi-experimental trial. International Journal of Infectious Diseases, 85, 16-21. https://doi.org/10.1016/j.jijid.2019.05.017

Marty Cooney, R., Manickam, N., Becherer, P., Harmon, L. S., Gregg, L., Farkas, Z., ... \& Shade, W. (2020). The 
use of $3.15 \%$ chlorhexidine gluconate $/ 70 \%$ alcohol hub disinfection to prevent central line-associated bloodstream infections in dialysis patients. British Journal of Nursing, 29(2), S24-S26. https://doi.org/10.12968/bjon.2020.29.2.S24

Dang, F. P., Li, H. J., Wang, R. J., Wu, Q., Chen, H., Ren, J. J., \& Tian, J. H. (2019). Comparative efficacy of various antimicrobial lock solutions for preventing catheter-related bloodstream infections: A network meta-analysis of 9099 patients from 52 randomized controlled trials. International Journal of Infectious Diseases, 87, 154-165. https://doi.org/10.1016/j.ijid.2019.08.017

Golestaneh, L., \& Mokrzycki, M. H. (2018). Prevention of hemodialysis catheter infections: Ointments, dressings, locks, and catheter hub devices. Hemodialysis International, 22(S2), S75-S82. https://doi.org/10.1111/hdi.12703

Hachem, R., Kanj, S., Hamerschlak, N., Saad, H., Assir, F. F., Mori, N., ... \& Raad, I. I. (2018). International experience with minocycline, EDTA and ethanol lock for salvaging of central line associated bloodstream infections. Expert review of medical devices, 15(6), 461-466. https://doi.org/10.1080/17434440.2018.1483237

Hill, J., \& Garner, R. (2020). Efficacy of 4\% tetrasodium ethylenediaminetetraacetic acid (T-EDTA) catheter lock solution in home parenteral nutrition patients: A quality improvement evaluation. The Journal of Vascular Access, 1129729820946916. https://doi.org/10.1177/1129729820946916

Kanaa, M., Wright, M. J., Akbani, H., Laboi, P., Bhandari, S., \& Sandoe, J. A. (2015). Cathasept line lock and microbial colonization of tunneled hemodialysis catheters: a multicenter randomized controlled trial. American Journal of Kidney Diseases, 66(6), 1015-1023. https://doi.org/10.1053/j.ajkd.2015.04.047

Labriola, L., \& Pochet, J. M. (2017). Any use for alternative lock solutions in the prevention of catheter-related blood stream infections?. The journal of vascular access, 18(1_suppl), S34-S38. https://doi.org/10.5301/jva.5000681

Leistner, R., Hirsemann, E., Bloch, A., Gastmeier, P., \& Geffers, C. (2014). Costs and prolonged length of stay of central venous catheter-associated bloodstream infections (CVC BSI): A matched prospective cohort study. Infection, 42(1), 31-36. https://doi.org/10.1007/s15010-013-0494-z

Liu, Y., Zhang, A. Q., Cao, L., Xia, H. T., \& Ma, J. J. (2013). Taurolidine lock solutions for the prevention of catheter-related bloodstream infections: a systematic review and meta-analysis of randomized controlled trials. PLoS One, 8(11), e79417. https://doi.org/10.1371/journal.pone.0079417

Liu, J., Wang, C. A., Zhao, H., Zhang, J., Ma, J., Hou, Y., \& Zou, H. (2015). Anticoagulant therapies versus heparin for the prevention of hemodialysis catheter-related complications: systematic review and meta-analysis of prospective randomized controlled trials. International journal of clinical and experimental medicine, $8(8)$, 11985. Retrieved from https://www.ncbi.nlm.nih.gov/pubmed/26550111

Mai, H., Zhao, Y., Salerno, S., Li, Y., Feng, Y., Ma, L., \& Fu, P. (2019). Citrate versus heparin lock for prevention of hemodialysis catheter-related complications: updated systematic review and meta-analysis of randomized controlled trials. International urology and nephrology, 51(6), 1019-1033. https://doi.org/10.1007/s11255-019-02150-0

Mandolfo, S., Anesi, A., Maggio, M., Rognoni, V., Galli, F., \& Forneris, G. (2020). High success rate in salvage of catheter-related bloodstream infections due to Staphylococcus aureus, on behalf of project group of Italian society of nephrology. The journal of vascular access, 21(3), 336-341. https://doi.org/10.1177/1129729819875323

Miller, L.M., Clark, E., Dipchand, C. et al. (2016). Hemodialysis Tunneled Catheter-Related Infections. Canadian Journal of Kidney Health and Disease, (3), 1-11. https://doi.org/10.1177/2054358116669129

Moghaddas, A., Abbasi, M. R., Gharekhani, A., Dashti-Khavidaki, S., Razeghi, E., Jafari, A., \& Khalili, H. (2015). Prevention of hemodialysis catheter-related blood stream infections using a cotrimoxazole-lock technique. Future microbiology, 10(2), 169-178. https://doi.org/10.2217/fmb.14.116

Moore, C. L., Besarab, A., Ajluni, M., Soi, V., Peterson, E. L., Johnson, L. E., ... \& Yee, J. (2014). Comparative effectiveness of two catheter locking solutions to reduce catheter-related bloodstream infection in hemodialysis patients. Clinical Journal of the American Society of Nephrology, 9(7), 1232-1239. https://doi.org/10.2215/CJN.11291113

Norris, L. B., Kablaoui, F., Brilhart, M. K., \& Bookstaver, P. B. (2017). Systematic review of antimicrobial lock 
therapy for prevention of central-line-associated bloodstream infections in adult and pediatric cancer patients. $\begin{array}{llll}\text { International journal of antimicrobial agents, } & \text { 50(3), 308-317. }\end{array}$ https://doi.org/10.1016/j.ijantimicag.2017.06.013

Padilla-Orozco, M., Mendoza-Flores, L., Herrera-Alonso, A., González, E. G., Ferman, J. L. G., Rodríguez-López, J. M., ... \& Camacho-Ortiz, A. (2019). Generalized and prolonged use of gentamicin-lock therapy reduces hemodialysis catheter-related infections due to gram negatives. Nephron, 143(2), 86-91. https://doi.org/10.1159/000500549

Pérez-Granda, M. J., Alonso, B., \&Rodríguez-Huerta, A. (2018), In vitro assessment of the anti-biofilm activity of ethanol alone and incombination with enoxaparin 60 IU. [Article in En, Spanish]. Enferm. Infecc. Microbiol. Clin, 36(10), 627-632. https://doi.org/10.1016/j.eimc.2017.11.007

Quirt, J., Belza, C., Pai, N., Clause, R. F., Markovic, F., Wong-Sterling, S., ... \& Wales, P. W. (2020). Reduction of Central Line-Associated Bloodstream Infections and Line Occlusions in Pediatric Intestinal Failure Patients Receiving Long-Term Parenteral Nutrition Using an Alternative Locking Solution, 4\% Tetrasodium Ethylenediaminetetraacetic Acid. Journal of Parenteral and Enteral Nutrition. https://doi.org/10.1002/jpen.1989

Raad, I., Chaftari, A. M., Zakhour, R., Jordan, M., Al Hamal, Z., Jiang, Y., ... \& Hachem, R. (2016). Successful salvage of central venous catheters in patients with catheter-related or central line-associated bloodstream infections by using a catheter lock solution consisting of minocycline, EDTA, and $25 \%$ ethanol. Antimicrobial agents and chemotherapy, 60(6), 3426-3432. https://doi.org/10.1128/AAC.02565-15

Rijnders, B., DiSciullo, G. J., Csiky, B., Rutkowski, B., Appelt, K., Cheronis, J., ... \& Fluck, R. (2019). Locking hemodialysis catheters with trimethoprim-ethanol-Ca-EDTA to prevent bloodstream infections: A randomized, evaluator-blinded clinical trial. Clinical Infectious Diseases, 69(1), 130-136. https://doi.org/10.1093/cid/ciy840

Sofroniadou, S., Revela, I., Kouloubinis, A., Makriniotou, I., Zerbala, S., Smirloglou, D., ... \& Iatrou, C. (2017). Ethanol combined with heparin as a locking solution for the prevention of catheter related blood stream infections in hemodialysis patients: A prospective randomized study. Hemodialysis International, 21(4), 498-506. https://doi.org/10.1111/hdi.12524

Song, T., \& Zhang, D. (2020). Evaluation on curative effects of ethylene diamine tetra-acetic acid chelation therapy in treating with atherosclerotic cardiovascular disease: A protocol for systematic review and meta-analysis. Medicine, 99(52). https://doi.org/10.1097/MD.0000000000023346

Sun, Y., Wan, G., \& Liang, L. (2020). Taurolidine lock solution for catheter-related bloodstream infections in pediatric patients: A meta-analysis. PloS one, 15(4), e0231110. https://doi.org/10.1371/journal.pone.0231110

Timsit, J. F., Bruneel, F., Cheval, C., Mamzer, M. F., Garrouste-Orgeas, M., Wolff, M., ... \& Carlet, J. (1999). Use of tunneled femoral catheters to prevent catheter-related infection: a randomized, controlled trial. Annals of internal medicine, 130(9), 729-735. https://doi.org/10.7326/0003-4819-130-9-199905040-00004

Torres-Viera, C., Thauvin-Eliopoulos, C., Souli, M., DeGirolami, P., Farris, M. G., Wennersten, C. B., ... \& Eliopoulos, G. M. (2000). Activities of taurolidine in vitro and in experimental enterococcal endocarditis. $\begin{array}{llll}\text { Antimicrobial agents and chemotherapy, 1720-1724. } & \text { 44(6), }\end{array}$ https://doi.org/10.1128/AAC.44.6.1720-1724.2000

Vercaigne, L. M., Allan, D. R., Armstrong, S. W., Zacharias, J. M., \& Miller, L. M. (2016). An ethanol/sodium citrate locking solution compared to heparin to prevent hemodialysis catheter-related infections: a randomized pilot study. The journal of vascular access, 17(1), 55-62. https://doi.org/10.5301/jva.5000486

Van Hulle, F., Bonkain, F., De Clerck, D., Aerden, D., Vanwijn, I., Tielemans, C., \& Wissing, K. M. (2019). Efficacy of urokinase lock to treat thrombotic dysfunction of tunneled hemodialysis catheters: A retrospective cohort study. The journal of vascular access, 20(1), 60-69. https://doi.org/10.1177/1129729818779549

Wang, J., Peng, X., Yin, W., Peng, Y., Liu, H., \& He, L. (2020). Eradication of microorganisms embedding in biofilm by a dose-dependent urokinase-based catheter lock solution in chronic hemodialysis patients. Blood purification, 49(4), 468-478. https://doi.org/10.1159/000505566

Winnicki, W., Herkner, H., Lorenz, M., Handisurya, A., Kikić, Ž., Bielesz, B., ... \& Sengoelge, G. (2018). Taurolidine-based catheter lock regimen significantly reduces overall costs, infection, and dysfunction rates of tunneled hemodialysis catheters. Kidney international, 93(3), 753-760. 
https://doi.org/10.1016/j.kint.2017.06.026

Yahav, D., Rozen-Zvi, B., Gafter-Gvili, A., Leibovici, L., Gafter, U., \& Paul, M. (2008). Antimicrobial lock solutions for the prevention of infections associated with intravascular catheters in patients undergoing hemodialysis: systematic review and meta-analysis of randomized, controlled trials. Clinical infectious diseases, 47(1), 83-93. https://doi.org/10.1086/588667

Yen, H. W., Yang, W. C., Tarng, D. C., Yang, C. Y., Chuang, C. L., Huang, L. J., ... \& Li, S. Y. (2016). Daptomycin antibiotic lock therapy for hemodialysis patients with Gram-positive bloodstream infections following use of tunneled, cuffed hemodialysis catheters: retrospective single center analysis. Hemodialysis International, 20(2), 315-320. https://doi.org/10.1111/hdi.12378

Zhao, T., Liu, H., \& Han, J. (2018). Ethanol lock is effective on reducing the incidence of tunneled catheter-related bloodstream infections in hemodialysis patients: a systematic review and meta-analysis. International urology and nephrology, 50(9), 1643-1652. https://doi.org/10.1007/s11255-018-1855-4

Zhao, Y., Li, Z., Zhang, L., Yang, J., Yang, Y., Tang, Y., \& Fu, P. (2014). Citrate versus heparin lock for hemodialysis catheters: a systematic review and meta-analysis of randomized controlled trials. American journal of kidney diseases, 63(3), 479-490. https://doi.org/10.1053/j.ajkd.2013.08.016

Zwiech, R., Adelt, M., \& Chrul, S. (2016). A Taurolidine-Citrate-Heparin Lock Solution Effectively Eradicates Pathogens From the Catheter Biofilm in Hemodialysis Patients. American journal of therapeutics, 23(2), e363-e368. https://doi.org/10.1097/MJT.0b013e31828d4610

\section{Copyrights}

Copyright for this article is retained by the author(s), with first publication rights granted to the journal.

This is an open-access article distributed under the terms and conditions of the Creative Commons Attribution license (http://creativecommons.org/licenses/by/4.0/). 